\title{
A Global View Beyond the Standard Model
}

\author{
Draft Version 0 \\ January 20, 2008
}

http://mlm.home.cern.ch/mlm/FlavLHC.html 
By 1973, the theoretical foundations of the Standard Model of fundamental interactions had been completed. In the decades that followed, new particles and phenomena predicted by the Standard Model were discovered in a dramatic series of experiments at laboratories around the world. This began with the discovery of the charm quark at SLAC and Brookhaven, predicted by Glashow, Illiopoulos and Maiani from flavor properties of the SM. The $\mathrm{W}$ and $\mathrm{Z}$ bosons were produced directly in experiments at CERN, and signals of energetic gluons were observed at DESY. Experiments eventually found a full third generation of fermions, culminating with the discovery of the top quark and tau neutrino at Fermilab.

During this same period, major theoretical advances made it possible to push the accuracy of Standard Model predictions. This allowed compelling tests of the SM at the level of radiative corrections, and to test the predictions of QCD in the confining domain. Thus experiments confirmed the quantum dynamics of the SM, and validated the CKM picture of flavor mixing and $\mathrm{CP}$ violation. While this process took a long time, and may have appeared frustrating to many to just achieve the confirmation of the "standard" theory, the outcome of these 30-odd years is now a cornerstone of our understanding of the natural world, occupying a deserved place next to Maxwell's electromagnetism, to relativity, and to quantum mechanics. The timescale and size of this enterprise, at the same time, gives us a benchmark for the magnitude of the efforts that may be required to go beyond the Standard Model to the next level of fundamental understanding.

New ideas and theories have been put forward in the attempt to understand great questions left unanswered by the Standard Model. These theories attempt to explain why nature needs both gravitational and gauge interactions, and why their energy scales are so different. They address the possible origins of matter-antimatter asymmetry, of particle masses, and why there are three families of fermions. A major theme of these new ideas is unification, beginning with grand unified theories and the introduction of supersymmetry. The continued elaboration of these ideas was accompanied and by the development of string theory, and by the introduction of the see-saw mechanism for neutrino masses, linking their smallness with the natural scale of grand unification. A second major theme has been the attempt to understand electroweak symmetry breaking at Terascale energies, involving both supersymmetry and frameworks with new forces, large extra-dimensions, and even theories with no higgs boson. While none of these new ideas has yet been confirmed, increasing evidence has built up that the SM itself cannot account for everything we observe. The discovery of neutrino masses, the matter antimatter asymmetry of the universe, and dark matter are in fact physics Beyond the Standard Model. The need to extend the SM is more than a purely theoretical exigency; it has become a compelling empirical requirement. Plausible and calculable BSM scenarios, merging the pragmatic need to explain recent discoveries with the desire to answer the theoretically inspired great questions, guide the setting of directions for the future of particle physics. 
The next step in the exploration of what's beyond the Standard Model will come from
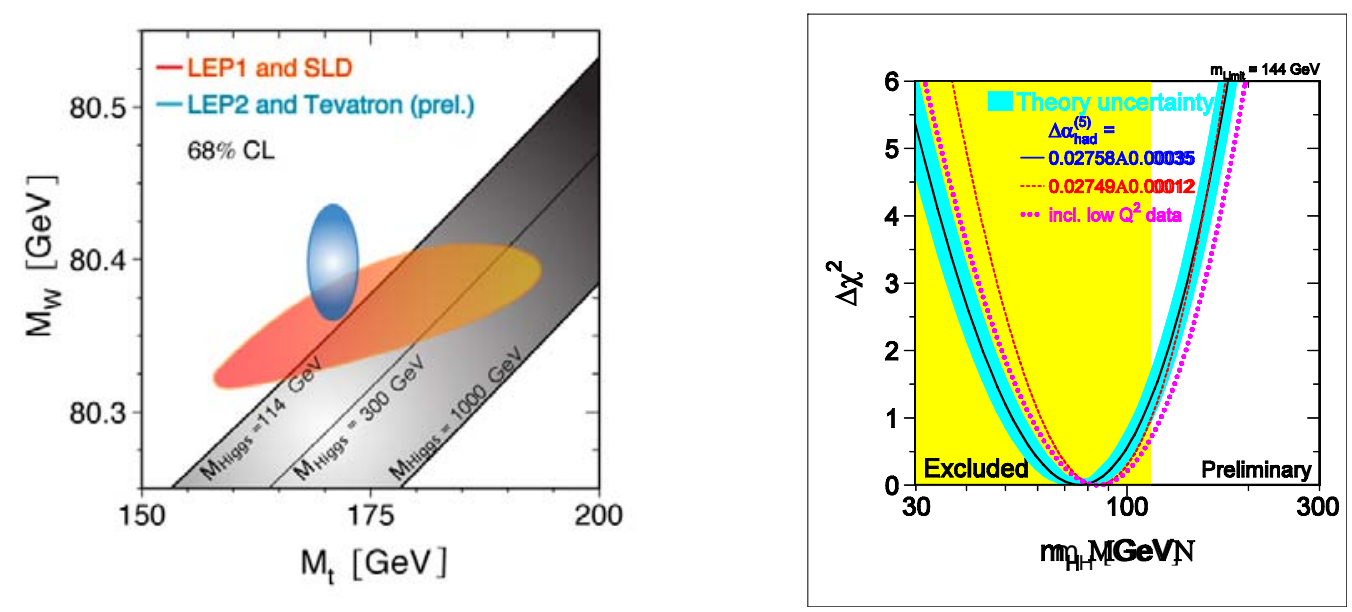

efforts to put into place the last missing piece of the SM itself, namely the higgs boson. The LHC, together with the Tevatron, will answer the question of whether there is a higgs particle or not. In the framework of the SM we could predict the higgs mass via other observables such as the $\mathrm{W}$ boson mass and the top quark mass, and we can accurately predict higgs production and decay rates. Deviations from the anticipated properties, a higgs heavier than the low-mass range preferred by the SM fits of electroweak observables, or complete lack of a higgs signal would all point to new physics. To which extent these departures can be understood within some of the new frameworks that have already been proposed, will depend on what we actually find. Since most of the competing BSM theories differ from each other in their formulation of the electroweak symmetry-breaking sector, the clarification of the origin of the higgs boson is an essential step in setting the scene for what is next. This step may take longer than the mere observation of higgs particle, and may not be achievable by the LHC alone, having to rely on progress of the energy-frontier facilities: LHC upgrades and a linear collider. For example, should supersymmetry be discovered at the LHC, a next big question of high-energy physics would become "how and why is supersymmetry broken?” The complexity of the validation of the SM will probably pale in comparison with the effort that will be required to study the supersymmetric sector, identify the supersymmetry breaking mechanism, understand the implications of supersymmetry for unification and cosmology, and prove the consistency of the new fundamental theory with accurate measurements and calculations. While the direct production of the expected exotic particles is the most desirable tool to analyze them, other experimental approaches will be necessary both to detect and decipher all of the new phenomena.

\section{The flavor of new physics}

Flavor phenomena have contributed to shaping the structure of the Standard Model as much as any other ingredient. The existence of flavor for quarks and leptons gives the SM its family and generation structure. Assembling quarks in electroweak doublets to suppress flavor-changing neutral currents with kaons led to the prediction of the charm quark. Kaon decays also led to the observation of CP violation, and to the CKM model, which in turn predicted the existence of a third generation. Mixing of neutral B mesons, similarly to the role played by neutral kaon mixing in getting the mass range for the 
charm quark, was the first experimental observation that correctly anticipated the large value of the top quark mass. The dramatic discovery of neutrino masses provided the first incontrovertible evidence that the SM is incomplete and may provide a window to grand unification. It is not surprising that several of the great questions of particle physics have flavor at their core, and it is reasonable to expect that flavor physics will continue playing a crucial role in the progress of the field.

There are several elements that directly associate LHC physics with flavor issues. In the absence of a higgs or some other mechanism of electroweak symmetry breaking, quark flavor effects would not even exist. All flavor phenomena in the SM are encoded by a handful of input parameters that lack any dynamical content. But when we move beyond the Standard Model, flavor phenomena can cover a much wider landscape and are even more strongly entangled with the dynamics of symmetry breaking. Flavor changing processes can be mediated by new particles such as charged higgses or supersymmetric partners. New flavors may appear, either in the form of new generations, or as exotic partners of standard quarks (such as Kaluza-Klein excitations in the case of extradimensional theories, or the T' and B' quarks in "little higgs" models). New sources of $\mathrm{CP}$ violation can arise from couplings of non-minimal higgs sectors, or of superpartners. With all of these new sources of flavor effects, the natural suppression of most flavor violating phenomena in the SM is put into jeopardy, and much larger effects are generally expected from new Terascale physics. In the context of BSM model building, this is a fundamental issue called "the flavor problem".

Equally important is the fact that in several BSM frameworks the parameters of flavor are not just arbitrary inputs but instead are the result of dynamics or symmetries of the underlying theory. Unified theories predict relations between the couplings of quarks and leptons. In supersymmetric models with neutrino masses, a mix of symmetry relations and dynamics connects neutrino mixing and flavor transitions in the charged-lepton sector. In extra-dimensional theories the family replicas can be understood as different branes on which fermions are bound to live, and mixings are tied to the relative positions of these branes in the extra dimensions. In supersymmetric theories, EWSB can be generated dynamically by the large value of the top quark mass, making EWSB, in some sense, a flavor-driven phenomenon. Finally, the numerical coincidence of the top mass value with the scale of EWSB is yet another mysterious hint of a possible direct connection between electroweak symmetry breaking and flavor.

These connections between symmetry breaking and flavor, as well as the flavor mysteries of neutrino masses and the matter antimatter asymmetry of the universe, very strongly suggest that flavor will play a key role in exploring the new physics landscapes unveiled by the LHC. Most new physics manifestations that we can envision will provide new sources of flavor phenomena, accentuating our need to address the flavor problem. The optimal approach to understanding flavor will depend on the details of what is discovered. It is sensible to expect, on the basis of the history of particle physics and of the explicit models of new physics available today, that experiments at the high-energy frontier and flavor experiments at the high-intensity frontier will provide complementary keystones in the coming phases of exploration of the laws of nature. The coincidence of many 
discoveries and measurements from many facilities will be needed to achieve a deeper understanding of the great questions of particle physics.

\section{The future of quark flavor experiments}

The study of the quark flavor sector is already highly advanced, as seen from the following figures, which show the consistency of a large number of a priori independent observables, all described by calculable combinations of just two CKM-matrix parameters $\bar{\rho}$ and $\bar{\eta}$ :
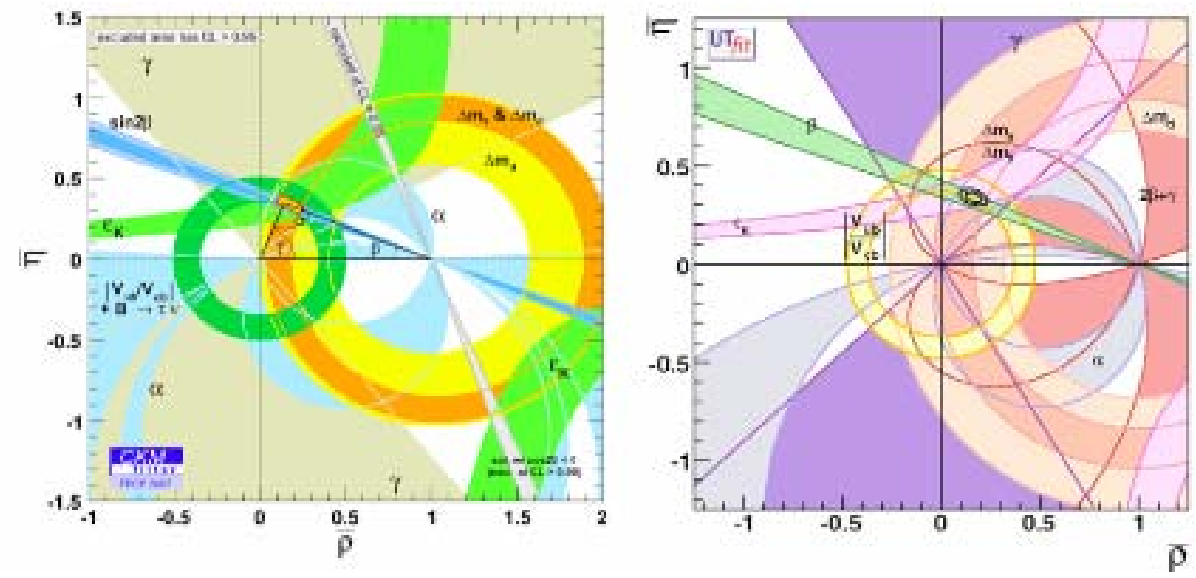

Just as precision measurements of electroweak observables agree very well with the theoretical predictions, the flavor structure prescribed by the SM appears to be very successful. But what is the origin of this success? Are the CKM parameters just arbitrary inputs, whose value was randomly selected by nature, or are they the result of some underlying dynamics? We ask similar questions about the gauge structure of the SM: are the relative strengths of the gauge forces independent parameters or are they related by some deeper causes? Here we believe we may have an answer, namely the idea of grand unification, which justifies the existence of several gauge couplings, and determines their values, as a result of the symmetry-breaking dynamics. Are there equivalent scenarios for the understanding of masses and flavor mixing? Could they emerge from some underlying dynamics? Is there a direct connection between the masses and mixings in the quark and lepton sectors? Neutrino masses and the matter antimatter asymmetry of the universe require new flavor phenomena beside those predicted by the SM: do these phenomena give related signatures involving quarks?

To address these questions we need to improve the accuracy of the measurements and of the theoretical predictions for quark flavor phenomena, looking for deviations from the SM picture. This approach will be vigorously pursued by the B-physics community, both at the LHC and at B factories. Several crucial tests, such as the measurement of the rare $B_{d, s}^{0} \rightarrow \mu^{+} \mu^{-}$decays, or of CP violation in the $B_{s}$ system, are still missing, but are within the reach of the forthcoming experiments. 
The decays $K_{L}^{0} \rightarrow \pi^{0} v \bar{v}$ and $K^{ \pm} \rightarrow \pi^{ \pm} v \bar{v}$, theoretically predicted with an uncertainty at the level of few percent within the SM, have a unique sensitivity to possible deviations from minimal flavor violation scenarios, where BSM physics enters with the observed CKM Yukawa coupling pattern. The minimal flavor violating framework can accommodate deviations in $K_{L}^{0} \rightarrow \pi^{0} v \bar{v}$ rates by factors of two-to-three while still respecting the stringent limits on new physics that would enter with generic couplings. The experimental measurement at the few percent level is therefore a must.

\section{The future of lepton flavor experiments}

Quark-lepton unification is as much an integral part of grand unification as is the unification of the gauge forces, underscoring once more the intimate connection and interplay between gauge and flavor phenomena. The existence of neutrino masses provides us with the possibility of probing quark-lepton dynamics at the GUT scale, something that would have never been possible had the neutrinos been massless.

The present goals of the neutrino program are very clear: to measure as accurately as possible the mass and mixing parameters, including the possible $\mathrm{CP}$ violating phase(s), the mass hierarchy, and determining the Dirac or Majorana nature of neutrino masses. These parameters represent fundamental constants of nature, and as such they have to be measured. We have no indication today of how precisely we need to know them, since there is no unique compelling theory of neutrino flavor. On the other hand, without a complete picture of the neutrino spectrum and mixings, including CP violation, we could not even start concretely testing possible underlying theories of leptonic flavor, and therefore their measurement has to be a priority.

The knowledge of the parameters of the neutrino sector is however not enough to answer all important questions. For example, $\mathrm{CP}$ violation in neutrino mixing could generate the matter antimatter asymmetry of the universe, but this is not determined conclusively just by the size of the CP violating parameters; it depends also on other new physics, such as the spectrum of the heavy partner neutrinos or supersymmetric partners. To understand whether CP violation in the neutrino sector can explain via leptogenesis the matter antimatter asymmetry of the universe, we have to learn more about the underlying theory. This requires establishing connections between flavor phenomena involving neutrinos, others involving quarks, and others yet to be discovered involving charged leptons. These connections can point to a common or unified origin of flavor phenomena, and give quantitative information about aspects of the theory that are otherwise inaccessible. A redundancy of inputs, capable of exposing small deviations from the simplest mixing picture, and probing the correlations between different observables predicted by different models, is an integral part of this program.

\section{The neutrino - muon - quark connection}

Studies of neutrino properties should be integrated with a more complete exploration of flavor phenomena with leptons. This should include both flavor-changing phenomena, 
such as muon to electron transitions, and CP-violating phenomena, such as electric dipole moments.

To illustrate the sort of interplay that may arise from a global approach to lepton flavor, consider the example of a supersymmetric grand unified $\mathrm{SO}(10)$ theory. Several structures for the mass terms are possible. For example:

$$
L_{m}=y_{i j}^{d, \ell} \mathbf{1 6}_{\mathrm{i}} \mathbf{1 6}_{\mathrm{j}} H_{d}+y_{i j}^{u, v} \mathbf{1 6}_{\mathrm{i}} \mathbf{1 6}_{\mathrm{j}} H_{u}+y_{i j}^{R} \mathbf{1 6}_{\mathrm{i}} \mathbf{1 6}_{\mathrm{j}} H_{R}^{126}
$$

where 16 $=\left(u_{L}, d_{L}, u^{c}, e^{c}\right)_{10}+\left(d^{c}, L\right)_{5}+N^{c}$ is the representation of $S O(10)$ containing all fields of a given generation (here decomposed in terms of SU(5) components). As suggested by the labels, the $\mathrm{H}_{\mathrm{d}}$ field gives a common mass to the down-type quarks and to the charged leptons; the $\mathrm{H}_{\mathrm{u}}$ field gives a common mass term to the up-type quarks and to mixing of the left- and right-handed neutrinos, and the $\mathrm{H}_{\mathrm{R}}$ field gives a Majorana mass to the righthanded, heavy neutrino $\mathrm{N}$. The neutrino mass matrix follows from the see-saw diagonalization,

$$
M_{v}=-\left(M^{u, v}\right)^{T}\left(M^{R}\right)^{-1} M^{u, v},
$$

where $M^{u, v}=y^{u, v}\left\langle H_{u}\right\rangle$ and $M^{R}=y^{R}\left\langle H_{R}\right\rangle$. With $y^{u, v}$ fixed by the knowledge of the CKM matrix, $y^{u, v}=V_{C K M} y_{\text {diag }}^{u} V_{C K M}{ }^{T}$, where $y_{\text {diag }}^{u}=\left(y_{u}, y_{c}, y_{t}\right)$; ; then the experimental knowledge about neutrino masses and mixings can then be turned into the determination of the righthanded neutrino mass matrix, $M_{R}$, resulting in the following spectrum ( $v_{1}$ being the lightest neutrino) :

$$
M_{R_{1}} \approx \frac{m_{t}^{2}}{4 m_{v_{1}}}, \quad M_{R_{2}} \approx \frac{m_{c}^{2}}{4 m_{v_{1}}}, \quad M_{R_{3}} \approx \frac{m_{u}^{2}}{2 m_{v_{1}}} .
$$

An additional interesting consequence of grand unification is that neutrino mixing leaks from the fermionic to the scalar sector, and via radiative effects, induces a mixing among the charged sleptons:

$$
\left(m_{\tilde{L}}^{2}\right)_{i j} \approx-\frac{3 m_{0}^{2}+A_{0}^{2}}{8 \pi^{2}} \sum_{k} y_{i k}^{v} y_{j k}^{v^{*}} \ln \frac{M_{G U T}}{M_{R_{k}}}
$$

where $m_{0}$ and $A_{0}$ are standard supersymmetry-breaking parameters. This mixing provides a seed for flavor-violating transitions among charged leptons, such as $\mu \rightarrow$ e $\gamma$ decay with a rate proportional to

$$
B(\mu \rightarrow e \gamma) \approx \frac{\alpha^{3}\left[\left(m_{\tilde{L}}^{2}\right)_{12}\right]^{2}}{G_{F}^{2} m_{S U S Y}^{8}} \tan ^{2} \beta
$$

With the previous choice of $y^{v}=y^{u}$, usually known as the CKM mixing case, $\left(m_{\tilde{L}}^{2}\right)_{12}$ results proportional to $V_{t d} V_{t s}$. Assuming instead $y^{v}$ proportional to the PMNS matrix, as can emerge from a different choice of higgs fields, $\left(m_{\tilde{L}}^{2}\right)_{12}$ would be proportional to $U_{\mu 3}$ $U_{e 3}$, a factor of about 140 larger than in the CKM case. The determination of $\mathrm{B}(\mu \rightarrow \mathrm{e} \gamma)$ could therefore be an important probe of flavor dynamics at the scale of unification. 
Finally, the coexistence of neutrinos and right-handed down-type quarks within the SU(5) multiplet coupling to the same higgs expectation value, points to a correlation between the neutrino mixing and the mixing of the $\tilde{b}_{R}$ and $\tilde{s}_{R}$ squarks, a mixing that could lead to observable contributions to $\mathrm{CP}$ violation in $B_{S} \rightarrow \varphi \psi$ or $B \rightarrow \varphi K_{S}^{0}$ decays, through diagrams such as:
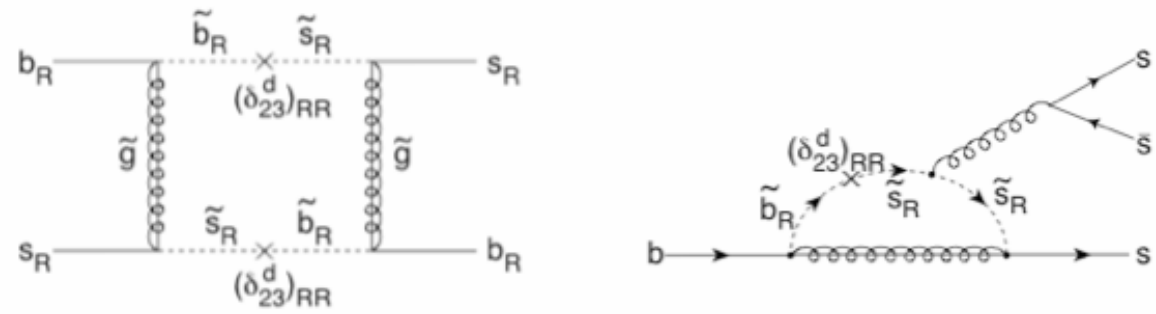

The above discussion shows therefore the existence of several spin offs of a description of neutrino masses in the context of a grand unified theory. The complete knowledge of the neutrino mass and mixing parameters is the necessary starting point for these studies: on one side it gives the crucial parameters that any model of neutrino masses and mixings must reproduce. On the other, it provides the inputs to evaluate the possible additional implications of new fundamental theory, making it possible to test the underlying theory at a deeper level. 